\title{
Measurement of apparent diffusion coefficient in discrimination of benign and malignant axillary lymph nodes
}

\author{
Ebru Yılmaz ${ }^{1 A, B, E}$, Berrin Erok $2 A, B, C, D, E, F, G$, Ali Önder Atca ${ }^{3 C, F}$ \\ 'Sarıer Hamidiye Etfal Education and Research Hospital, İstanbul, Turkey \\ ${ }^{2}$ Cihanbeyli State Hospital, Konya, Turkey \\ ${ }^{3}$ Altınbas University School of Medicine Bahcelievler Medical Park Hospital, İstanbul, Turkey
}

\begin{abstract}
Purpose: We aimed to determine the contribution of the apparent diffusion coefficient (ADC) value in the detection of axillary lymph node metastasis.

Material and methods: Breast magnetic resonance of 58 patients, performed in the radiology clinic of our hospital between 2015 and 2017 were examined retrospectively, and 43 lymph nodes in 43 patients were included in the study. They were evaluated morphologically on T1W and T2W sequences, and the lymph nodes showing rounded shape, focal or diffuse cortical thickness of more than $3 \mathrm{~mm}$, and partial or total effacement of fatty hilum were included in the study. Subsequently, their ADC values were measured.

Results: There were 43 lymph nodes, 20 of which were malignant and 23 of which were benign. While the mean ADC value of malignant axillary lymph nodes was $0.74910^{-3} \mathrm{~mm}^{2} / \mathrm{s}(0.48-1.342)$, it was $0.98210^{-3} \mathrm{~mm}^{2} / \mathrm{s}(0.552-1.986)$ for benign lymph nodes. When the ADC cut-off value was taken as $\leq 0.753 \times 10^{-3} \mathrm{~mm}^{2} / \mathrm{s}$, its discrimination power between benign and malignant axillary lymph nodes was as follows: sensitivity - 60\%; specificity - 91.3\%; accuracy - $76.7 \%$; positive predictive value $-85.7 \%$; and negative predictive value $-72.4 \%$.

Conclusions: There was no significant difference between mean ADC value of 12 lymphadenopathies (LAP) associated with inflammatory breast diseases (granulomatous mastitis and acute suppurative mastitis) and mean ADC value of metastatic lymph nodes. However, the ADC value of lymph nodes showing thickened cortex due to systemic inflammatory diseases was over 1 , and there was a statistically significant difference when compared with metastatic lymph nodes.
\end{abstract}

Key words: axillary LAP, breast cancer, diffusion-weighted imaging (DWI), apparent diffusion coefficient (ADC).

\section{Introduction}

The most important prognostic factor in breast cancer is whether or not the axillary lymph nodes include metastasis, and if so, the number of metastatic lymph nodes. When the five-year survival for patients with negative axillary lymph nodes is $82.8 \%$, it decreases proportionally with the number of metastatic nodes [1-4]. The presence of metastatic lymph nodes alters the treatment modality and the surgical method. Therefore, preoperative diagnosis of nodal metastasis is extremely important. Axillary dissection is an invasive procedure which may be associated with com- plications reducing the quality of life $[5,6]$. The sentinel lymph node (SLN) procedure emerged to reduce unnecessary axillary lymphadenectomy, and the associated morbidity was shown to be successful in patients with breast cancer; however, when the literature was analysed, it was seen that the rate of false negativity was higher than expected. For this reason, preoperative radiological diagnosis of nodal metastasis with high accuracy is very important. To that end, the main imaging modality is ultrasound (US) imaging. On US the sensitivity and specificity is increased by using morphological criteria as a base in distinction of benign and malignant lymph nodes. In differentiation of

Correspondence address:

Dr. Berrin Erok, Cihanbeyli State Hospital, Radiology, Konya, Turkey, e-mail: drberrinerok@hotmail.com

Authors' contribution:

A Study design · B Data collection · C Statistical analysis · D Data interpretation · E Manuscript preparation · F Literature search · G Funds collection 
benign and malignant breast lesions, by making post-contrast kinetic analysis in addition to morphological criteria in conventional magnetic resonance (MR) imaging, the sensitivity is increased to approximately $86-100 \%$, but the specificity does not show a similarly high rate. Therefore, in order to increase specificity, additional sequences were sought $[7,8]$. It was shown that measurement of the apparent diffusion coefficient (ADC) obtained from diffusion-weighted sequences (DWI) added to conventional MR increases specificity and provides more accurate differentiation of benign and malignant breast lesions [7-11]. However, evaluation of axillary lymphadenopathies (LAP) with DWI is still under study. In our study, we aimed to determine the contribution of the ADC value to morphological criteria evaluated with conventional MR imaging in the detection of nodal metastasis.

\section{Material and methods}

Breast MR of 58 patients, performed in the radiology clinic of our hospital between 2015 and 2017, were examined retrospectively, and the lymph nodes having focal or diffuse cortical thickness more than $3 \mathrm{~mm}$ were included in the study.

Approval was obtained from our hospital's Ethics Board (date: 04.04.2018, decision: 20), and informed written consent was obtained from each patient.

At first, the lymph nodes were evaluated morphologically on T1W and T2W images, and the lymph nodes showing rounded shape, focal or diffuse cortical thickness more than $3 \mathrm{~mm}$, and partial or total effacement of fatty hilus were included in the study. After that, without knowing about primary breast diseases, follow-up period, and pathological results, they were evaluated on DWI sequences and their ADC values were measured. The lymph nodes of 12 patients, which were apparent on conventional MR sequences, were not visualised on DWI possibly due to insufficient resolution especially for the nodes smaller than $5 \mathrm{~mm}$ in diameter and motion artefact, so they were excluded from the study. Additionally, because three patients had received neoadjuvant chemotherapy, they were also excluded from the study. As a result, a total of 43 lymph nodes in 43 patients were included in the study, and the mean age was $44.42 \pm 12.15$ years (26-73). Thirty-two out of 43 patients had been diagnosed histopathologically by surgical excisional biopsy or thick needle biopsy, and the remaining 11 lymph nodes had been evaluated firstly as benign and during a one-year follow-up period at six-month intervals they were accepted as benign as they decreased in size and the cortical thickness returned to normal.

\section{Magnetic resonance imaging}

All examinations were performed with 1.5 Tesla (T) MRI equipment (GE Signa HDx, GE Medical Systems, USA) using an eight-channel phased array breast surface coil. Care was taken to perform the breast MRI of pre-menopausal women between the $5^{\text {th }}$ and $15^{\text {th }}$ days of the menstrual cycle. Conventional contrast MRI images were obtained with the following technique: axial fat-suppressed T2-weighted turbo spin-echo (repetition time/echo time; TR/TE), $4500 \mathrm{~ms} / 97 \mathrm{~ms}, 330 \mathrm{~mm}$ field of view (FOV), number of excitations (NEX): 1, matrix, $384 \times 512$; slice thickness of $3 \mathrm{~mm}$ with a $1 \mathrm{~mm}$ intersection gap and fat-suppressed T1-weighted (TR/TE, 720 ms/20 ms; 330 mm FOV, NEX: 2 , matrix, $320 \times 320$; slice thickness of $3 \mathrm{~mm}$ ), and fat-suppressed 3D T1-weighted images $(4.3 \mathrm{~ms} / 1.4 \mathrm{~ms}$; flip-angle, $12^{\circ}$; a FOV of $320 \mathrm{~mm}$; matrix, $307 \times 512$; signal average 1 ; slice thickness, $1.5 \mathrm{~mm}$ fast low-angle shot [FLASH]) with and without contrast. Sagittal fat-supressed 3D T1-weighted images were obtained six minutes after contrast material injection in addition to axial dynamic contrast-enhanced images. The contrast agent (Dotarem, Laboratoire Guerbet, Roissy, France) was administered at $0.2 \mathrm{mmol} /$ $\mathrm{kg}$ via automatic syringe, followed by $15-20 \mathrm{cc}$ of saline for homogeneous distribution of the contrast substance. DWI echo-planar images (TR/TE 8500/70, FOV of $330 \mathrm{~mm}$, matrix $192 \times 192$, NEX: 1 , sectional thickness $4.5 \mathrm{~mm}$ with a $1 \mathrm{~mm}$ intersection gap) were obtained in the axial plane before contrast administration. DWI were obtained by diffusion gradients between 0 and $1000 \mathrm{~s} / \mathrm{mm}^{2}$ b-values.

\section{Apparent diffusion coefficient analysis}

An ADC map was automatically constructed in a commercially available workstation. Mean ADC values of all lesions were automatically measured by using these maps according to the formula $\mathrm{ADC}=(\operatorname{lnS} 0-\ln S) / \mathrm{b}$ (signal intensity values are measured as $\mathrm{S} 0$ at $\mathrm{b}=0 \mathrm{~s} / \mathrm{mm}^{2}$ and $\mathrm{S}$ at $\mathrm{b}=1000 \mathrm{~s} /$ $\mathrm{mm}^{2}$ ). Measurements were performed by placing a region of interest (ROI) of $0.5 \mathrm{~mm}$ diameter on lesions. ADC measurements were performed on enhancing or solid parts of the lesions identified in conventional sequences. The ROI did not include normal parenchymal tissue, or haemorrhagic or necrotic areas. One radiologist with experience in breast MRI evaluated the images and performed measurements of $\mathrm{ADC}$ and $\mathrm{rADC}$ values. A minimum of three ADC measurements were performed, and the lowest $\mathrm{ADC}$ value was accepted. The ADC value of the contralateral breast at the same level was also measured after ADC measurements were completed for the lesion and ROI of the same diameter was used. The $\mathrm{rADC}$ was calculated as the mass ADC value divided by the ADC value of adjacent parenchyma. In addition, the maximum diameters of the tumours on sagittal and axial planes were measured and the mean of these values were expressed as "mean maximum size".

\section{Statistical analysis}

SPSS 25.0 (IBM Corparation, Armonk, New York, United States) software was used for the analysis of variables. The conformity of normal distribution of data was evalu- 
ated by Shapiro-Wilk test and homogeneity of variances by Levene. While comparing benign and malign groups with each other according to age-independent-samples $t$-test with Bootstrap results were used, and for long and short diameter and ADC comparison Mann-Whitney $U$ test with Monte Carlo simulation technique was used. In the comparison of hilux situation with malignity Fisher exact test was verified by Exact results. Odds ratio with confidence interval was used in order to show how many more times the hilum situation affects malignity. The relationship between the classification with cut-off value calculated according to ADC variable and the real classification was defined by examination of sensitivity, specificity, positive predictivity, negative predictivity, diagnostic accuracy, and ROC (receiver operating curve) analysis. Quantitative variables were presented as mean \pm SD (standard deviation), minimum-maximum and median (minimum/maximum), and categorical variables were presented as $n(\%)$. Variables were examined in confidence interval of $95 \%$, and the significance level for the study was set as $p<0.05$.

\section{Results}

In our study there were 43 lymph nodes, 20 of which were malignant and 23 of which were benign. The mean age of the patients with benign lymph nodes was $39.52 \pm$ 9.54 years (26-61), and the mean age of the patients with malignant lymph nodes was $50.05 \pm 12.60$ years (27-73). The mean age of the malignant group was significantly higher than that of the benign group $(p=0.005)$ (Figure 1 ).

While the mean length of the long diameter of malignant lymph nodes was $15 \mathrm{~mm}$ (7-34), it was $14 \mathrm{~mm}$ (7-30) for benign lymph nodes. There was no statistically significant difference between the two groups $(p=0.492)$.

The mean length of the short diameter of malignant lymph nodes was $9 \mathrm{~mm}$ (5-23), and it was $8 \mathrm{~mm}$ (5-14) for benign lymph nodes. There was no statistically significant difference between the two groups $(p=0.539)$.

The mean ADC value of malignant axillary lymph nodes was $0.74910^{-3} \mathrm{~mm}^{2} / \mathrm{s}(0.48-1.342)$, and it was $0.98210^{-3} \mathrm{~mm}^{2} / \mathrm{s}(0.552-1.986)$ for benign lymph nodes. There was a statistically significant difference between the two groups $(p<0.001)$ (Figure 2$)$.

When the ADC cut-off value was taken as $\leq 0.753 \times$ $10^{-3} \mathrm{~mm}^{2} / \mathrm{s}$, its discriminative power between benign and malignant axillary lymph nodes was as follows: sensitivity $-60 \%$, specificity $-91.3 \%$, accuracy $-76.7 \%$, positive predictive value $-85.7 \%$, and negative predictive value $72.4 \%$ (Figures 3 and 4 ).

\section{Discussion}

Breast cancer is the most common type of cancer in women, and despite advances in diagnosis and treatment, it is the second most common cause of cancer-related deaths

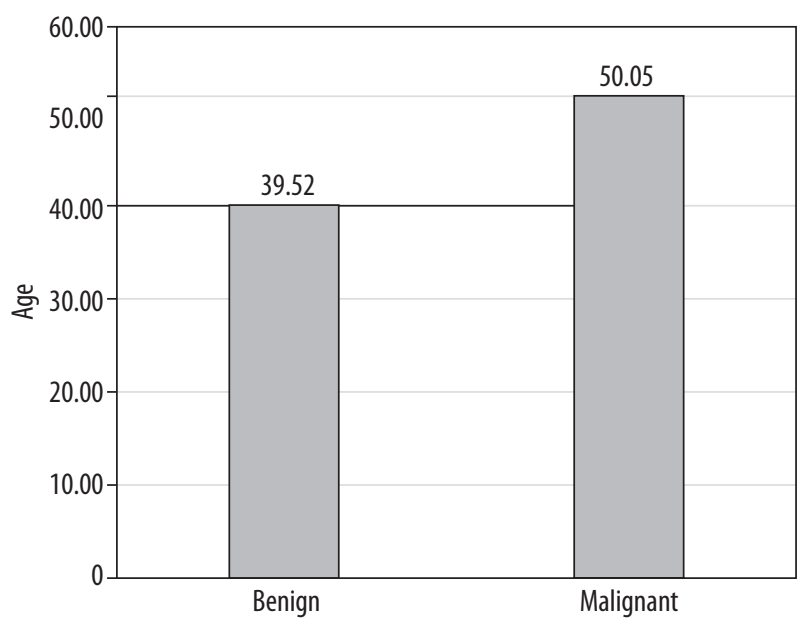

Figure 1. Age distribution

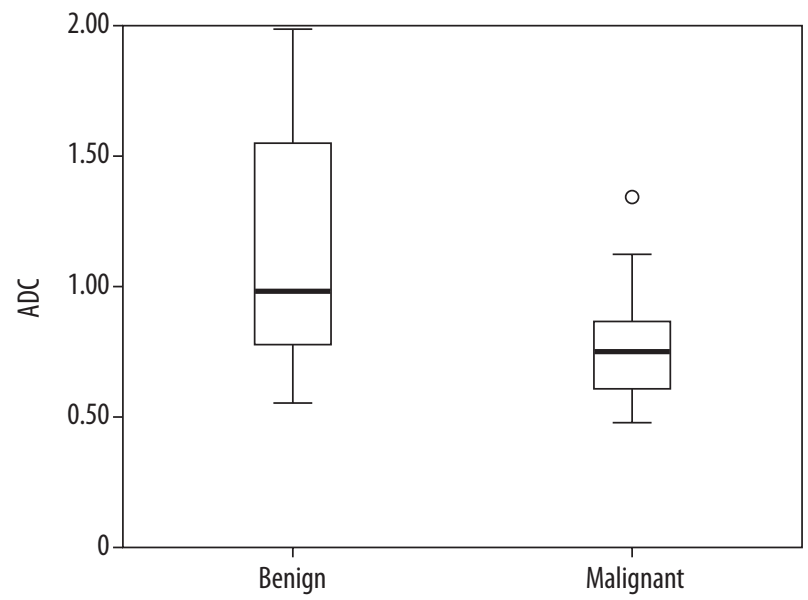

Figure 2. Apparent diffusion coefficient value of benign/malignant lymphadenopathies

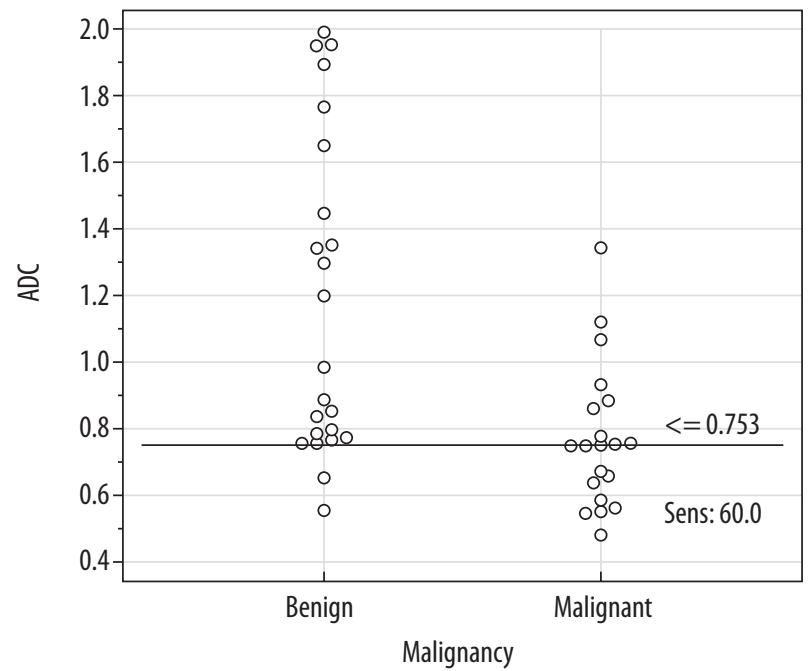

Figure 3. Apparent diffusion coefficient cut off value

after lung cancer [12]. Being the most important prognostic factor, the presence of malignant axillary lymph nodes in breast cancer plays a critical role in staging, treatment planning, and long-term survival $[1,13]$. On mammography, the axillary region can be evaluated partially. Classi- 


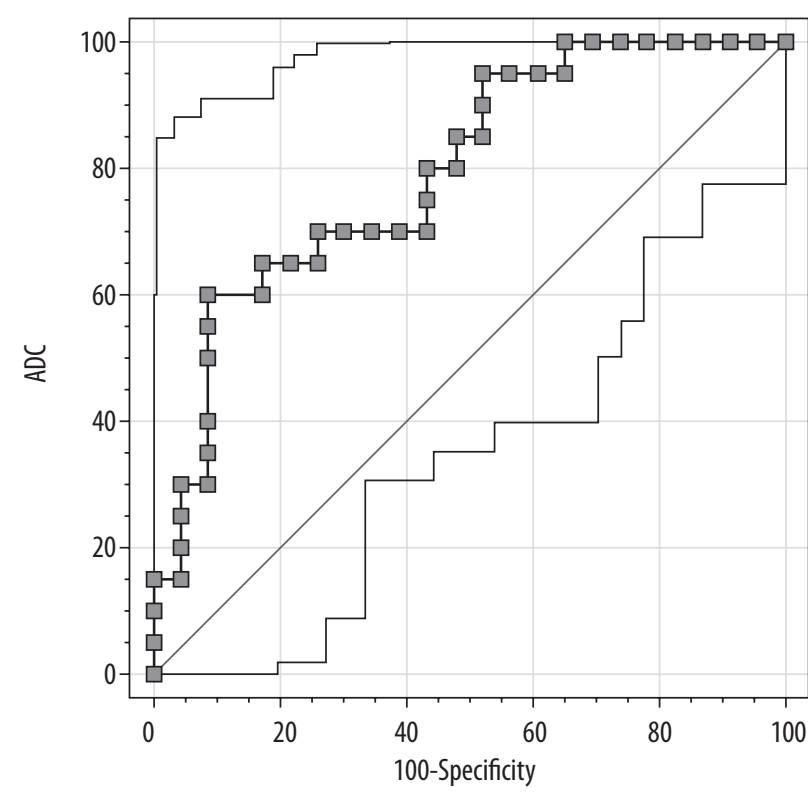

Figure 4. Sensitivity and specificity

cally, normal axillary lymph nodes are seen as oval-shaped opacities having a maximum diameter less than $2 \mathrm{~cm}$ and radiolucent notch. Increased size, number, density, and effacement of the radiolucent hilum cause suspicion of being pathological. It is known that the best modality in the evaluation of axilla is US. However, the sensitivity and specificity of US in the detection of axillary metastatic lymph nodes are variable and contradictory [14]. US is operator dependent and is insufficient in the evaluation of deeply located lymph nodes [15]. Biopsy is the gold standard modality in the evaluation of axillary LAP. However, it is both an invasive procedure and has a high false negativity rate due to possible wrong sampling [16]. In recent years, in order to increase sensitivity and specificity, DWI has been added to conventional breast MR. In many studies, the benefit of DWI in the differentiation of malignant and benign breast lesions as well as following the response to treatment has been shown, and so it is included in routine MR protocols in many centres [17-20]. The evaluation of axillary LAP by DWI is still under study and is controversial.

When water molecules are placed in a container, they undergo Brownian motion without any obstacles. This motion, which is not restricted by anything, is called free diffusion [21]. The DWI value shows the level of diffusion of water molecules in tissues. The diffusion of water in tissues was restricted basically by cell membranes and cellularity of the tissue. When the cell membranes are destroyed, water molecules move freely or when the cellularity increases, which means the number of cell membranes increases (in tumoral processes) the motion of water molecules restricted [11]. In order to measure diffusion in tissues, before and after $180^{\circ} \mathrm{RF}$ pulses, two gradients having equal force and time (diffusion gradients) are applied in EPI sequences. These gradients should be strong enough to determine the movement dependent on diffusion. The diffusion weight factor (b value) shows the extent to which the sequence is sensitive to the diffusion. When the $b$ value is low, perfusion and the T2 time affect diffusion, and the diffusion weight of the sequence decreases. The perfusion of malignant tumours is markedly higher than that of the benign ones, and so at low $b$ values the $\mathrm{ADC}$ of malignant lesions were measured as higher than it is. However, if the b value is equal to or higher than $600 \mathrm{~s} / \mathrm{mm}^{2}$, the effect of perfusion is shown to be mini$\mathrm{mal}$ and can be ignored [22]. Because, when the $b$ value increases the spatial resolution decreases significantly, and when the $b$ value decreases then the perfusion effect appears, in our study the $b$ value was taken as $1000 \mathrm{~s} / \mathrm{mm}^{2}$.

$\mathrm{ADC}$ is the mathematical expression of diffusion obtained as a result of marking the signal losses on the map after the application of diffusion gradients. In order to obtain an ADC map two imagings with and without diffusion gradient are performed. The ADC value is the negative logarithm of the signal ratios of these two images [23]. The ADC value of free water molecules at $37^{\circ} \mathrm{C}$ is reported to be $3.0 \times 10^{-3} \mathrm{~mm}^{2} / \mathrm{s}$. This value is taken as the reference point, and it is the value at which the diffusion is maximum. When there is restriction to diffusion the ADC value decreases [11].

In most of the studies conducted in the literature, patients with diagnosis of invasive breast carcinoma were taken as a study group and by comparing ADC values of axillary lymph nodes, its contribution to differentiation of malignant and benign lymph nodes was investigated [24-26]. In the study conducted by Kim et al. [24] the ADC value for metastatic lymph nodes was $0.91 \times 10^{-3} \mathrm{~mm}^{2} / \mathrm{s}$ and it was $1.27 \times 10^{-3} \mathrm{~mm}^{2} / \mathrm{s}$ for benign lymph nodes; in the study of Razek et al. [25] the ADC value for metastatic lymph nodes was $1.08 \times 10^{-3} \mathrm{~mm}^{2} / \mathrm{s}$ and it was $1.15 \times 10^{-3} \mathrm{~mm}^{2} / \mathrm{s}$ for benign lymph nodes; in the study of Chung et al. [26] the ADC value for metastatic lymph nodes was $0.69 \times 10^{-3}$ $\mathrm{mm}^{2} / \mathrm{s}$ and it was $1.04 \times 10^{-3} \mathrm{~mm}^{2} / \mathrm{s}$ for benign lymph nodes; and in the study conducted by Hazanzadeh et al. [27] the ADC value for metastatic lymph nodes was 0.824 $\times 10^{-3} \mathrm{~mm}^{2} / \mathrm{s}$ and it was $1.098 \times 10^{-3} \mathrm{~mm}^{2} / \mathrm{s}$ for benign lymph nodes. In these studies, the ADC values were significantly lower in metastatic lymph nodes. In the detection of metastatic axillary lymph nodes the accuracy rate of the ADC value was reported to be $80 \%$ in the study of Scaraleno et al. [28]. In the study of Choi et al. the malignant possibility of the axillary lymph nodes having cortical thickness more than $3 \mathrm{~mm}$ was found to be 4.4 times higher than that of the axillary lymph nodes with cortical thickness less than $3 \mathrm{~mm}$, and in ultrasound evaluation the cortical thickness was defined as the most accurate sign in detection of metastasis [29]. In addition to that, in the study of Scaranelo et al. a higher rate of being associated with malignity was established for axillary lymph nodes with cortical thickness more than $3 \mathrm{~mm}$ [28].

By taking as references these data from the literature, we evaluated all axillary lymph nodes with cortical thickness more than $3 \mathrm{~mm}$ without any consideration about 
primary breast disease, and then their ADC values were measured without knowing about primary breast disease, follow-up period, and pathology results [29].

Morphologically the most common cause of malignant-looking LAP is inflammatory pathologies. In the study conducted by Wang et al. [30] no significant morphological difference was established between the lymph nodes that underwent a change due to metastasis and inflammatory pathologies. In our study, there were 12 LAPs associated with inflammatory breast diseases (pathologically proven nine granulomatous mastitis and three acute suppurative mastitis), and their mean ADC value was $0.868 \times 10^{-3} \mathrm{~mm}^{2} / \mathrm{s}$. No statistically significant difference was found when compared with mean ADC value of metastatic axillary lymph nodes. However, in the lymph nodes with thickened cortex associated with systemic inflammatory diseases, the value of ADC was over $1 \mathrm{~mm}^{2} / \mathrm{s}$ and there was a statistically significant difference when compared with mean ADC values of metastatic axillary lymph nodes.

In the study conducted by Wang et al. [30] and Vandacavaye et al. [31] the signal of both the malignant and inflammatory lymph nodes was hyperintense on DWI, and it was specified that they were not differentiated from each other by using DWI signal. In also our study, all the lymph nodes showed hyperintensity on DWI in accordance with the literature.

The main limitations of our study are: the limited number of patients, exclusion of lymph nodes with diameter less than $5 \mathrm{~mm}$, even if they have rounded shape and effaced hilum because of not being visualised on DWI, and the validity of the cut-off DC value only for the imaging protocols performed with the $b$ values defined for our device.

\section{Conclusions}

ADC values of axillary lymph nodes secondary to systemic inflammatory processes have been found over $1 \times$ $10^{-3} \mathrm{~mm}^{2} / \mathrm{s}$ and were significantly higher when compared with the ADC values of metastatic LAP (Figure 5), and the malignant-benign distinction could be made. However, in the axillary LAP associated with inflammatory pathologies of the breast, like granulomatous mastitis and acute suppurative mastitis (Figure 6), there was no significant difference between their ADC values, and the malignant-benign distinction could not be made. We believe that this information will be obtained more accurately and reliably in studies conducted with larger patient series.

\section{Disclosure}

The authors declare no conflicts of interest.

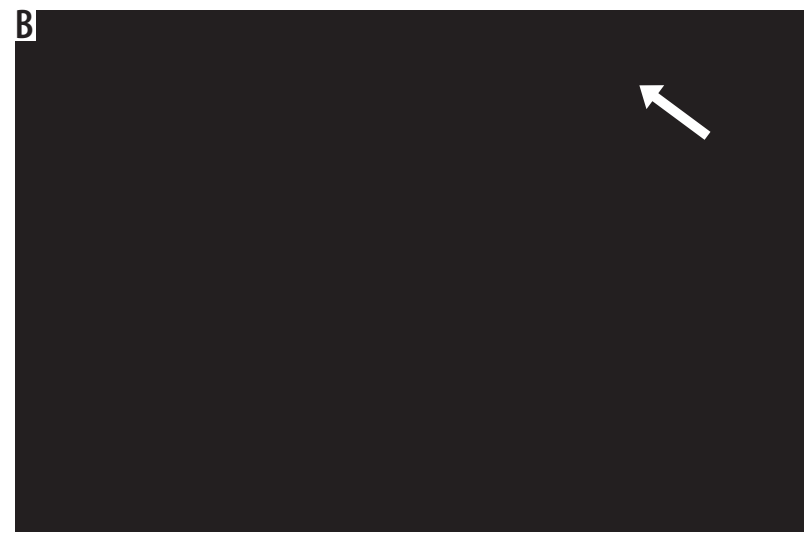

Figure $5.10 \times 7 \mathrm{~mm}$ right axillary lymphadenopathies with diffuse cortical thickening, seen as hyperintense on diffusion-weighted imaging and hypointense on apparent diffusion coefficient (restricted diffusion) (ADC: $0.584 \times 10^{-3} \mathrm{~mm}^{2} / \mathrm{s}$ ) (pathology: carcinoma metastasis from ipsilateral breast)
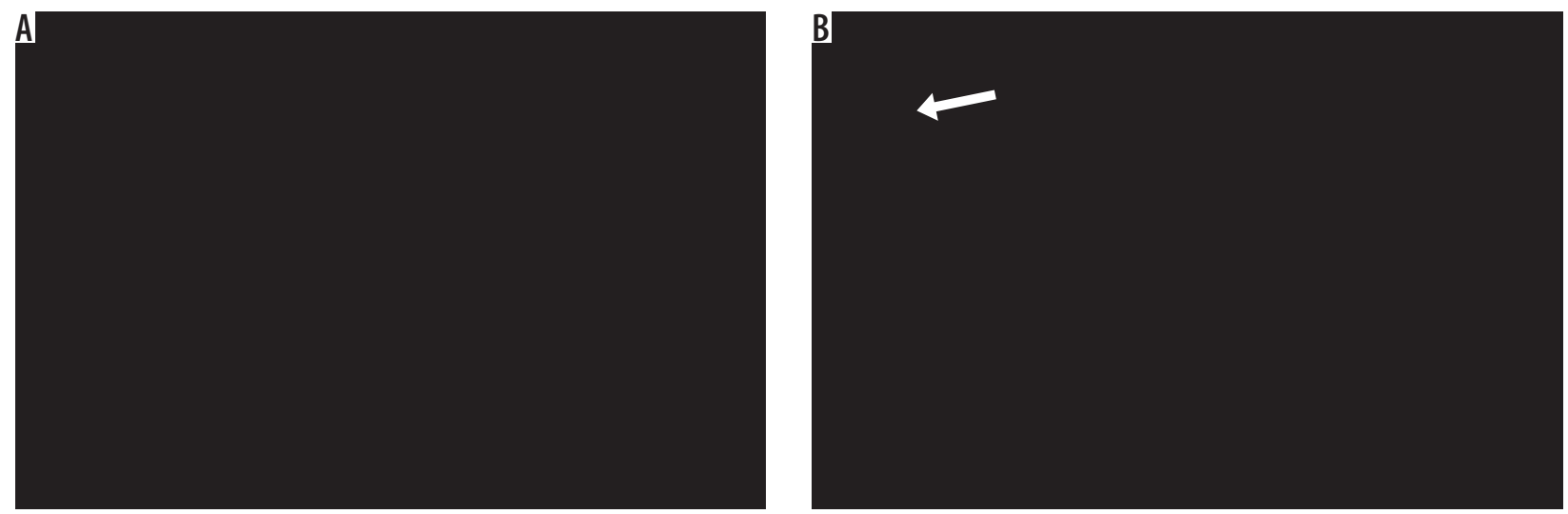

Figure $6.14 \times 12 \mathrm{~mm}$ sized left axillary lymphadenopathies with rounded shape, seen as hyperintense on diffusion-weighted imaging and hypointense on apparent diffusion coefficient (restricted diffusion) (ADC: $0.768 \times 10^{-3} \mathrm{~mm}^{2} / \mathrm{s}$ ) (pathology: reactive hyperplasia associated with acute suppurative mastitis in the ipsilateral breast) 


\section{References}

1. Fisher B, Bauer M, Wickerham DL, et al. Relation of number of positive axillary nodes to the prognosis of patients with primary breast cancer. An NSABP update. Cancer 1983; 52: 1551-1557.

2. Fuster D, Duch J, Paredes P, et al. Preoperative staging of large primary breast cancer with [18F] fluorodeoxyglucose positron emission tomography/computed tomography compared with conventional imaging procedures. J Clin Oncol 2008; 26: 4746-4751.

3. Krammer J, Wasser K, Schnitzer A, et al. Axillary lymph node characterization in breast cancer patients using magnetic resonance mammography: a prospective comparative study with FDG PET-CT and healthy women. Eur J Radiol 2013; 82: 2194-2198.

4. Li C, Meng S, Yang X, et al. The value of T2* in differentiating metastatic from benign axillary lymph nodes in patients with breast cancer - a preliminary in vivo study. PLoS One 2014; 9: e84038.

5. Martelli G, Boracchi P, De Palo M, et al. A randomized trial comparing axillary dissection to no axillary dissection in older patients with T1N0 breast cancer results after 5 years of follow-up. Ann Surg $2005 ; 242: 1-6$

6. Baltzer PA, Dietzel M, Burmeister HP, et al. Application of MR mammography beyond local staging: is there a potential to accurately assess axillary lymph nodes? Evaluation of an extended protocol in an initial prospective study. AJR Am J Roentgenol 2011; 196: W641-647.

7. Blackledge MD, Leach MO, Collins DJ, Koh DM. Computed diffusion-weighted MR imaging may improve tumor detection. Radiology 2011; 261: 573-581.

8. El Khouli RH, Jacobs MA, Mezban SD, et al. Diffusion-weighted imaging improves the diagnostic accuracy of conventional $3.0-\mathrm{T}$ breast MR imaging. Radiology 2010; 256: 64-73.

9. Woodhams R, Matsunaga K, Kan S, et al. ADC mapping of benign and malignant breast tumors. Magn Reson Med Sci 2005; 4: 35-42.

10. Şahin C, Arıbal E. The role of apparent diffusion coefficient values in the differential diagnosis of breast lesions in diffusion-weighted MRI. Diagn Interv Radiol 2013; 19: 457-462.

11. Woodhams R, Ramadan S, Stanwell P, et al. Diffusion-weighted imaging of the breast: principles and clinical applications. Radiographics 2011; 31: 1059-1084.

12. Huang W, Fisher PR, Dulaimy K, et al. Detection of breast malignancy: diagnostic MR protocol for improved specificity. Radiology 2004; 232: 585-591

13. Silverstein MJ, Skinner KA, Lomis TJ. Predicting axillary nodal positivity in 2282 patients with breast carcinoma. World J Surg 2001; 25: 767-772.

14. Alvarez S, Anorbe E, Alcorta P, et al. Role of sonography in the diagnosis of axillary lymph node metastases in breast cancer: a systematic review. AJR Am J Roentgenol 2006; 186: 1342-1348.

15. Cools-Lartigue J, Meterissian S. Accuracy of axillary ultrasound in the diagnosis of nodal metastasis in invasive breast cancer: a review. World J Surg 2012; 36: 46-54.

16. Mullen R, Purdie CA, Jordan LB, et al. Can additional histopathological examination of ultrasound-guided axillary lymph node core biopsies improve preoperative diagnosis of primary breast cancer nodal metastasis? Clin Radiol 2013; 68: 704-707.
17. Kul S, Cansu A, Alhan E, et al. Contribution of diffusion-weighted imaging to dynamic contrast-enhanced MRI in the characterization of breast tumors. AJR Am J Roentgenol 2011; 196: 210-217.

18. Kuroki-Suzuki S, Kuroki Y, Nasu K, et al. Detecting breast cancer with non-contrast MR imaging: combining diffusion-weighted and STIR imaging. Magn Reson Med Sci 2007; 6: 21-27.

19. Yilmaz E, Bayramoglu Z, Kartal MG, et al. Stromal fibrosis: imaging features with diagnostic contribution of diffusion-weighted MRI. Br J Radiol 2018; 91: 20170706.

20. Wang XH, Peng WJ, Tan HN, et al. Value of diffusion weighted imaging (DWI) in evaluating early response to neoadjuvant chemotherapy in locally advanced breast cancer. Zhonghua Zhong Liu Za Zhi 2010; 32: 377-381.

21. Koh DM, Collins DJ. Diffusion-weighted MRI in the body: applications and challenges in oncology. AJR Am J Roentgenol 2007; 188: 1622-1635.

22. Baron P, Dorrius MD, Kappert P, et al. Diffusion-weighted imaging of normal fibroglandular breast tissue: influence of microperfusion and fat suppression technique on the apparent diffusion coefficient. NMR Biomed 2010; 23: 399-405.

23. Stejskal EO, Taner JE. Spin diffusion measurements: spin-echo in the presence of a time dependent field gradient. J Chem Phys 1965; 42: 288-292.

24. Kim EJ, Kim SH, Kang BJ, et al. Diagnostic value of breast MRI for predicting metastatic axillary lymph nodes in breast cancer patients: diffusion-weighted MRI and conventional MRI. Magn Reson Imaging 2014; 32: 1230-1236.

25. Razek AA, Lattif MA, Denewer A, et al. Assessment of axillary lymph nodes in patients with breast cancer with diffusion-weighted MR imaging in combination with routine and dynamic contrast MR imaging. Breast Cancer 2016; 23: 525-532.

26. Chung J, Youk JH, Kim JA, et al. Role of diffusion-weighted MRI: predicting axillary lymph node metastases in breast cancer. Acta Radiol 2014; 55: 909-916.

27. Hasanzadeh F, Faeghi F, Valizadeh A, Bayani L. Diagnostic value of diffusion weighted magnetic resonance imaging in evaluation of metastatic axillary lymph nodes in a sample of Iranian women with breast cancer. Asian Pac J Cancer Prev 2017; 18: 1265-1270.

28. Scaranelo AM, Eiada R, Jacks LM, et al. Accuracy of unenhanced MR imaging in the detection of axillary lymph node metastasis: study of reproducibility and reliability. Radiology 2012; 262: 425-434.

29. Choi YJ, Ko EY, Han BK, et al. High-resolution ultrasonographic features of axillary lymph node metastasis in patients with breast cancer. Breast 2009; 18: 119-122.

30. Wang J, Liao Q, Zhang Y, et al. Differential diagnosis of axillary inflammatory and metastatic lymph nodes in rabbit models by using diffusion-weighted umaging: compared with conventional magnetic resonance imaging. Korean J Radiol 2012; 13: 458-466.

31. Vandecaveye V, De Keyzer F, Vander Poorten V, et al. Head and neck squamous carcinoma: a value of diffusion weighted MR imaging for nodal staging. Radiology 2009; 251: 134-146. 